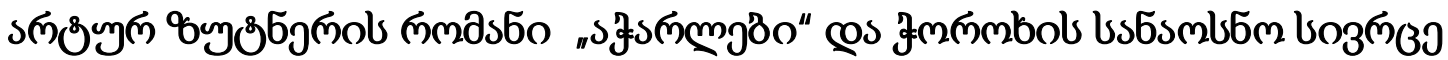

\author{
Rmbsmsdo asmbss

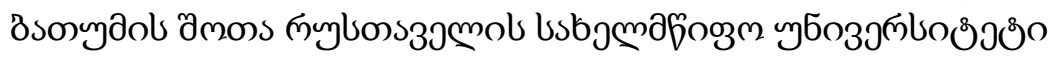

https://doi.org/10.52340/idw.2021.525

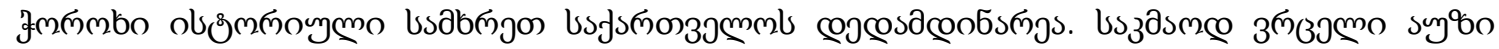

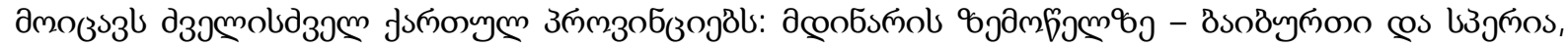

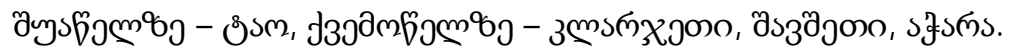

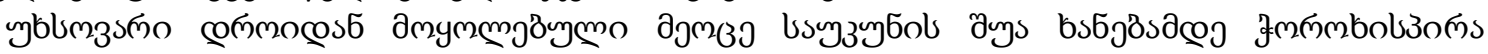

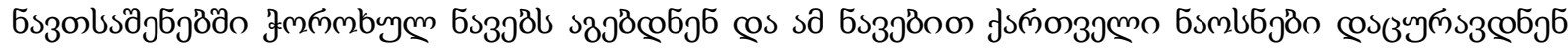

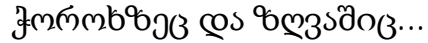

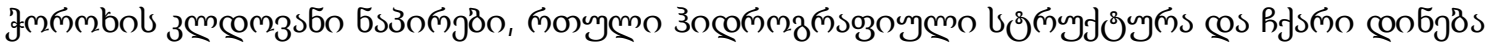

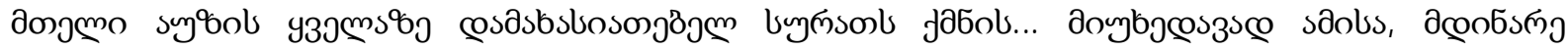

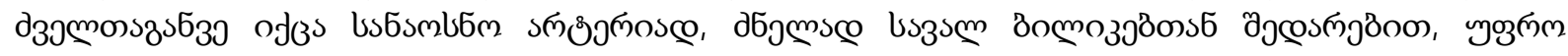

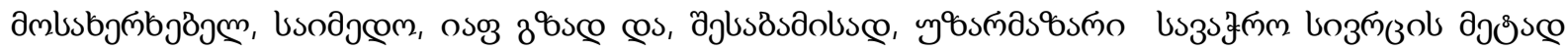

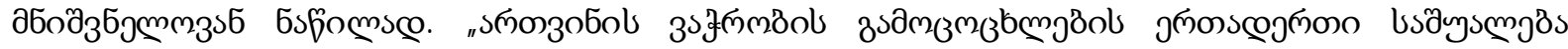

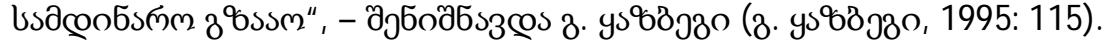

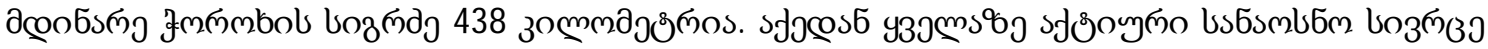

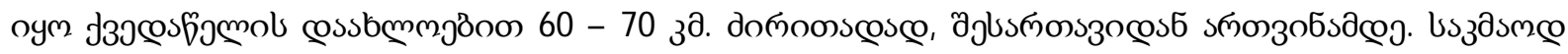

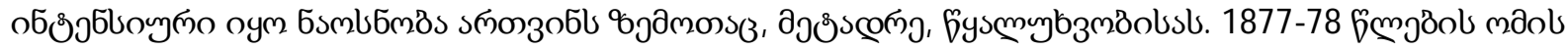

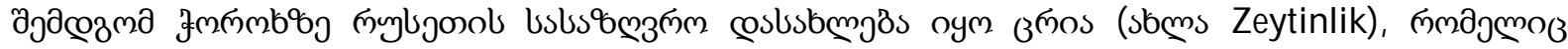

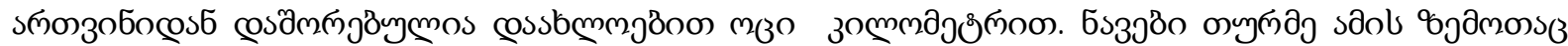

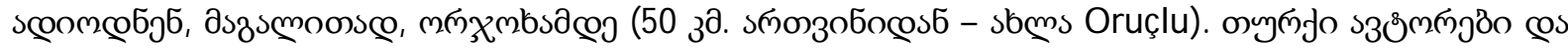

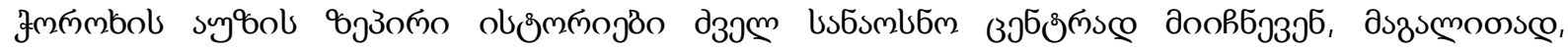

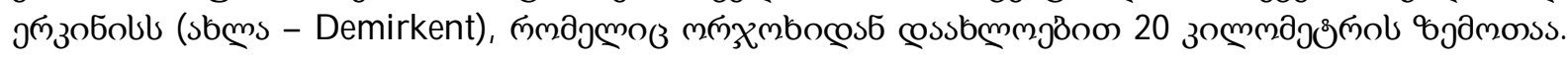

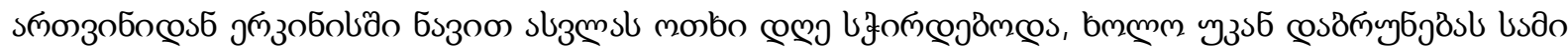

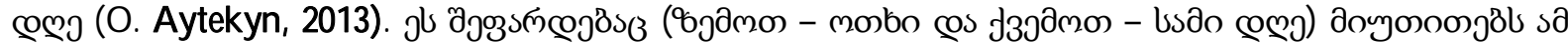

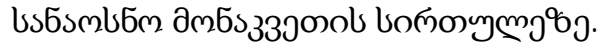

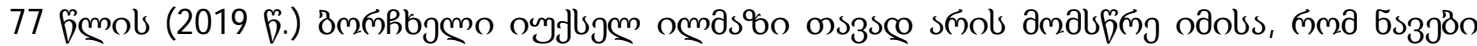

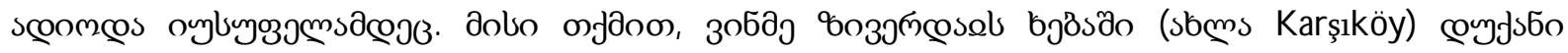

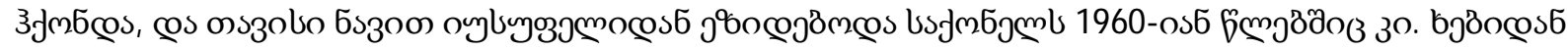

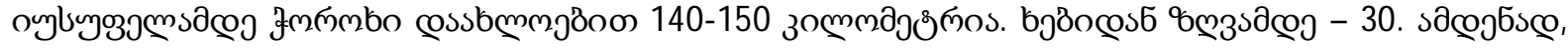

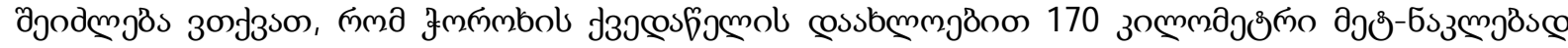

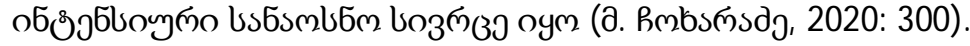

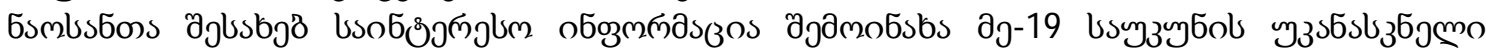

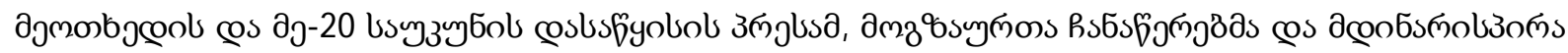

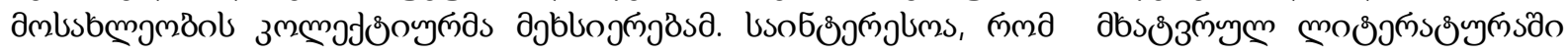

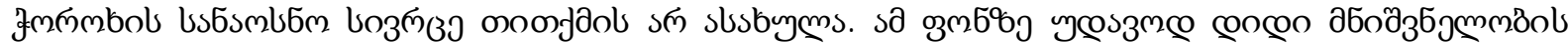

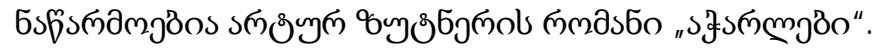

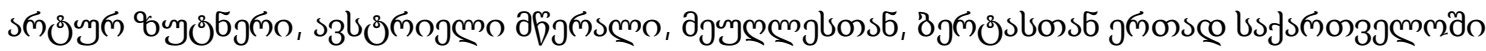

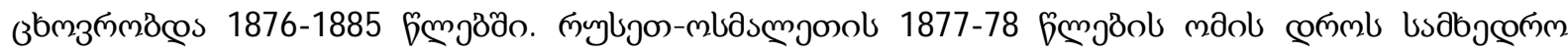

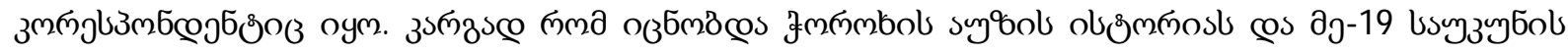

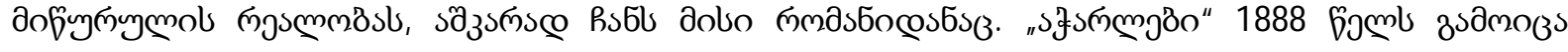




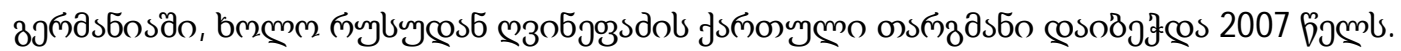

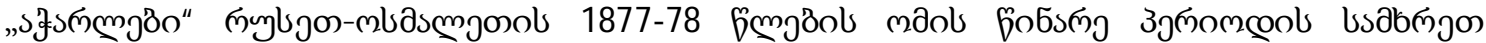

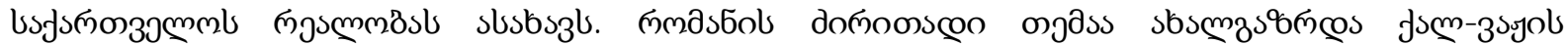

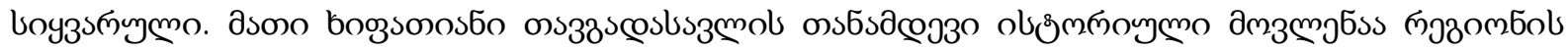

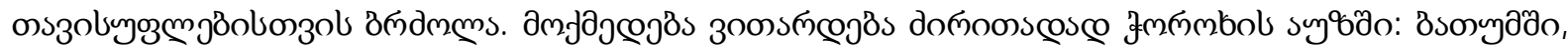

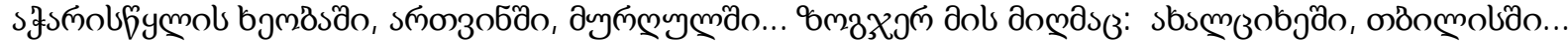

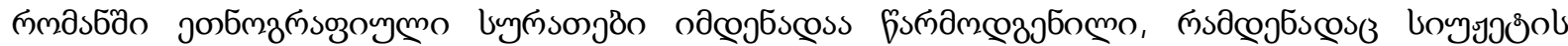

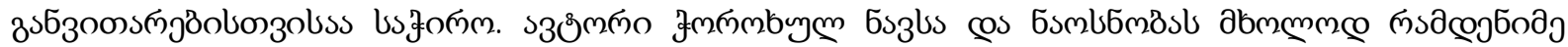

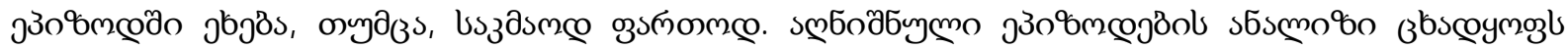

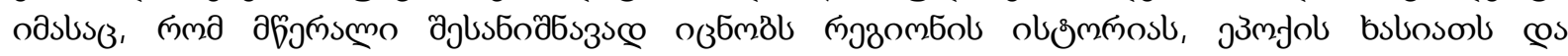

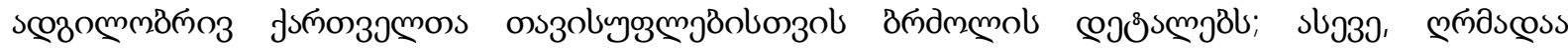

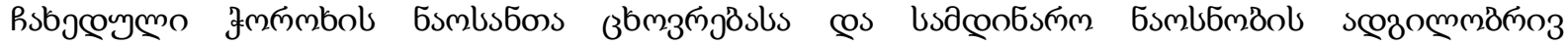

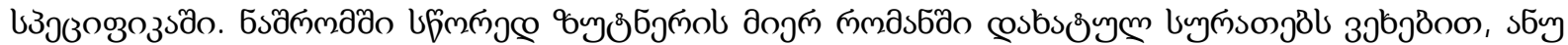

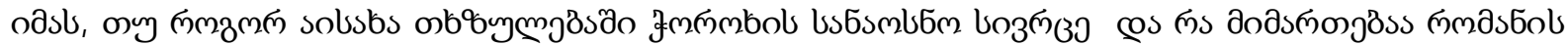

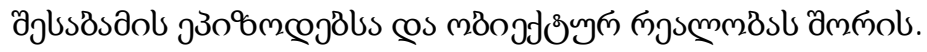

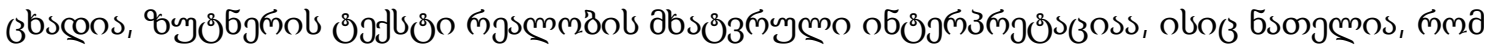

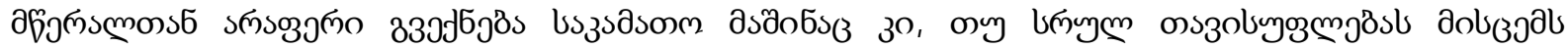

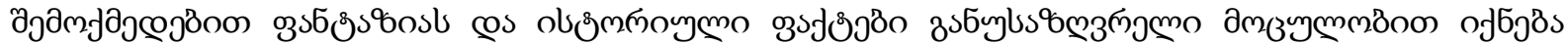

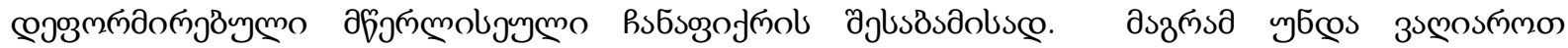

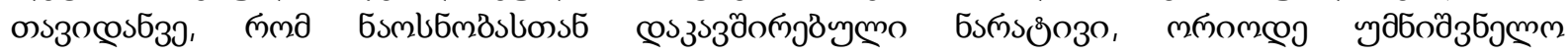

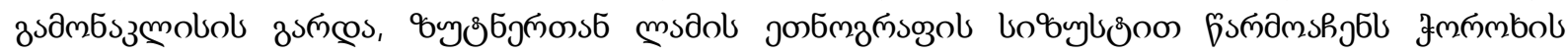

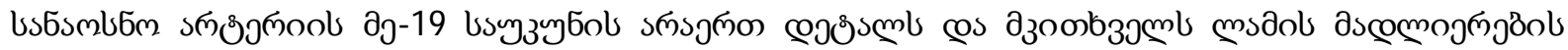

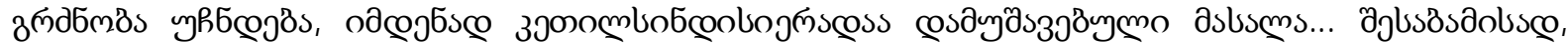

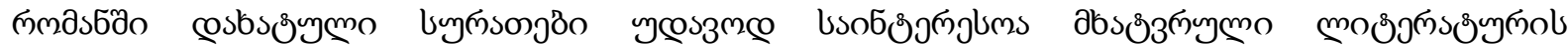

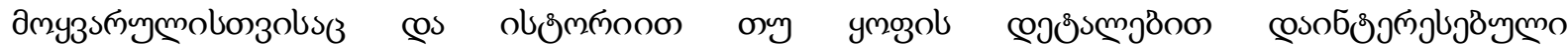
azoob 3 jomolsonzolsiz...

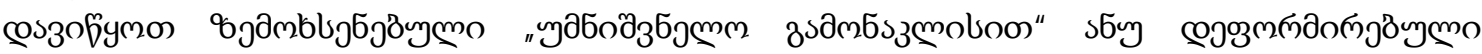

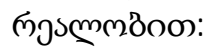

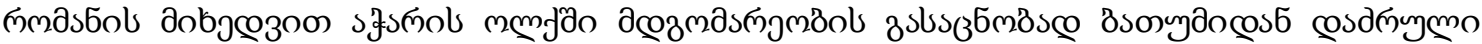

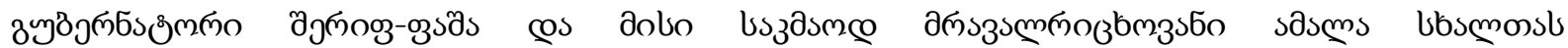

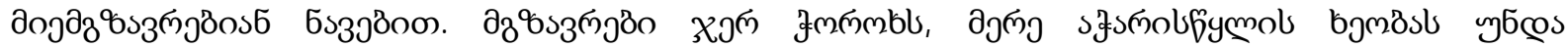

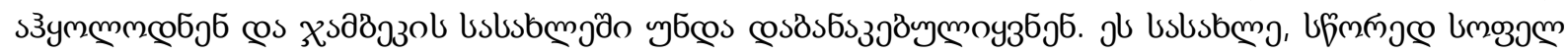

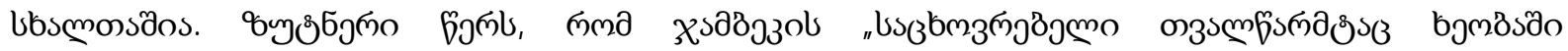

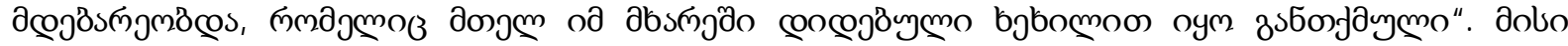

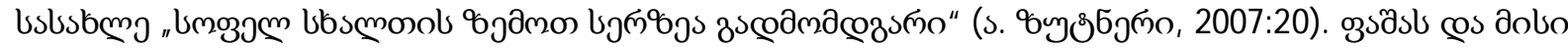

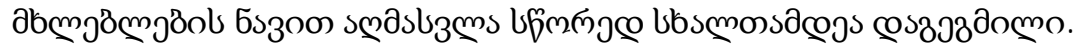

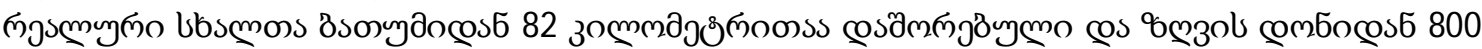

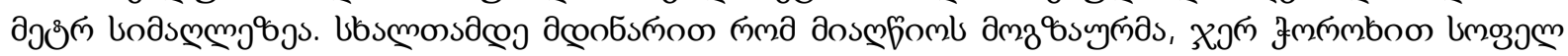

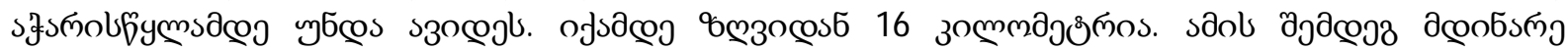

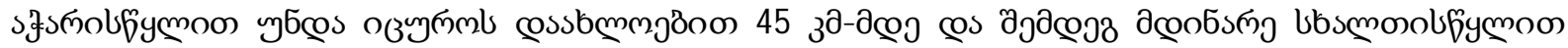

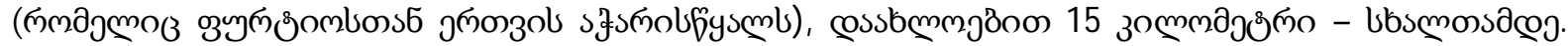

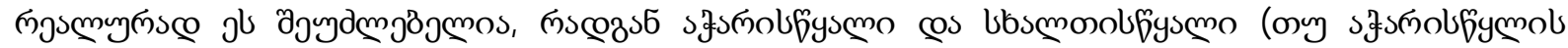

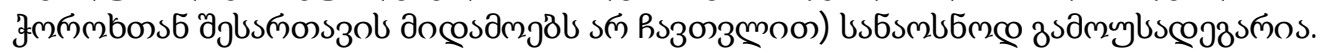

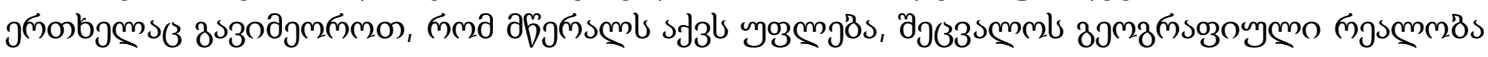

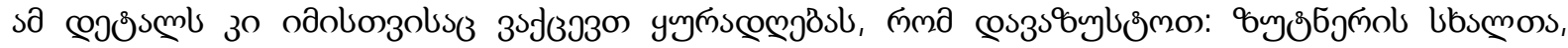

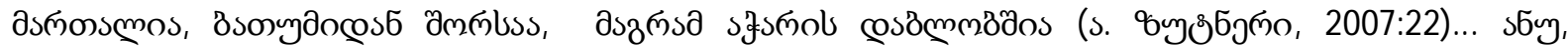

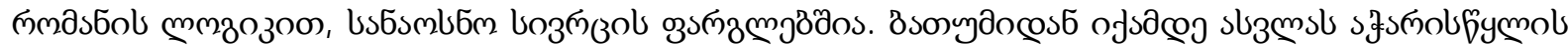

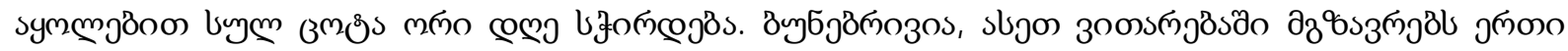

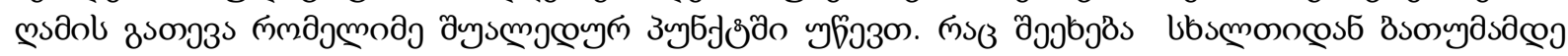

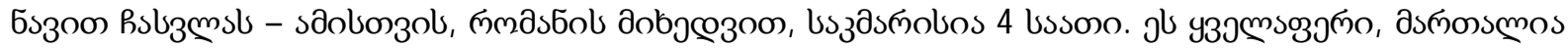




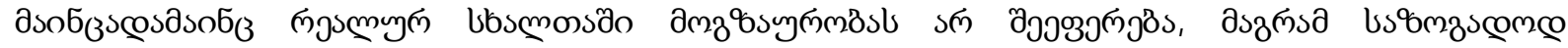

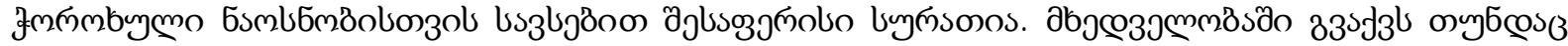

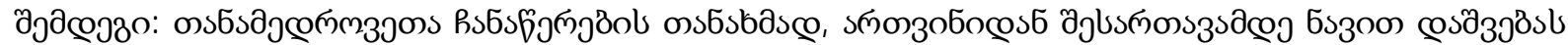

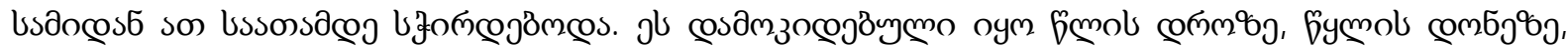

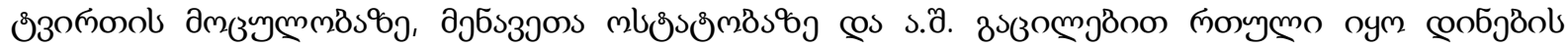

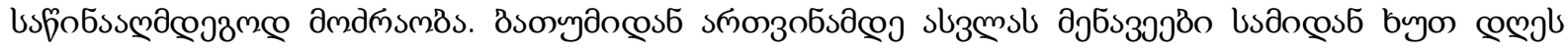

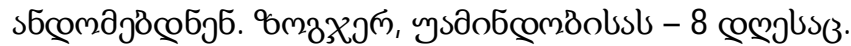

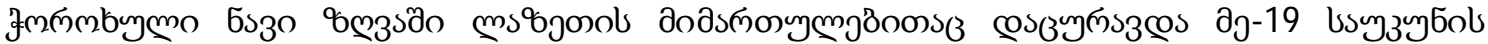

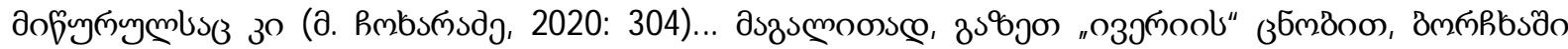

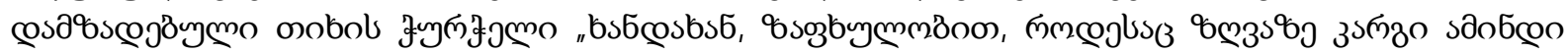

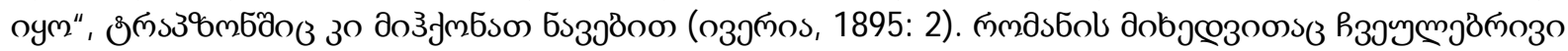

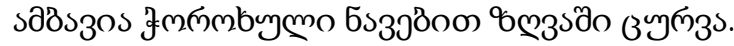

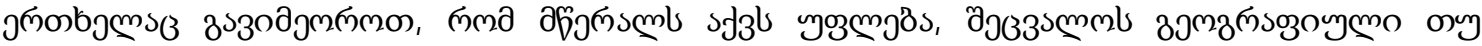

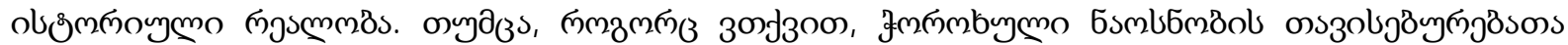

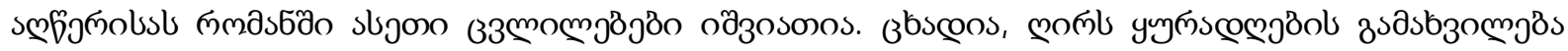

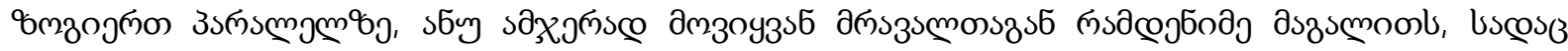

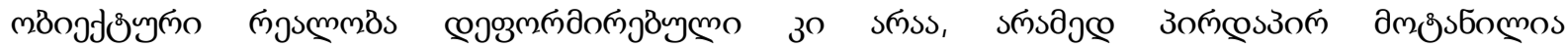

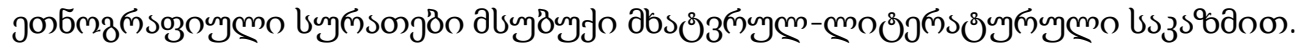

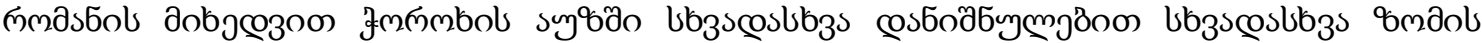

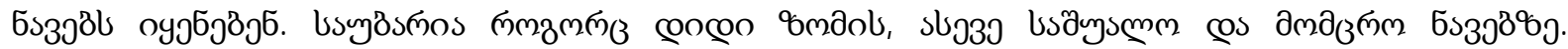

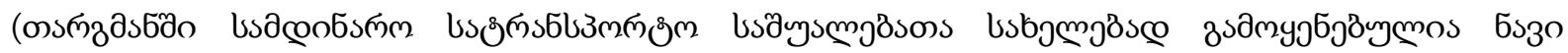

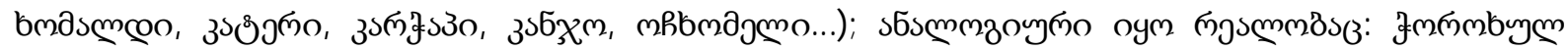

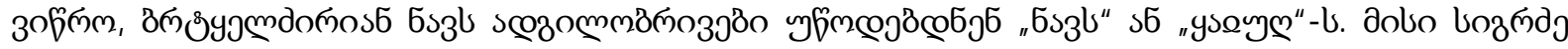

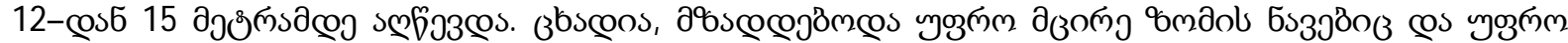

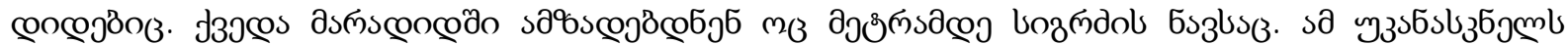

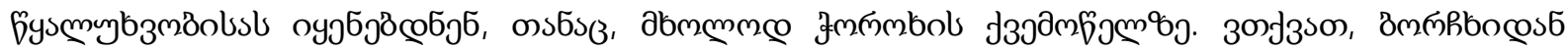

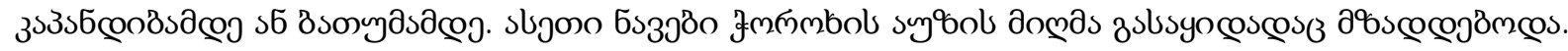

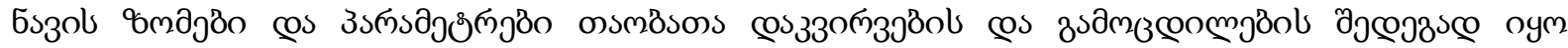

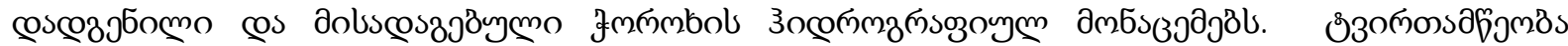

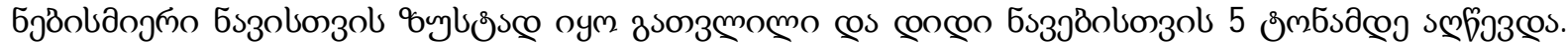

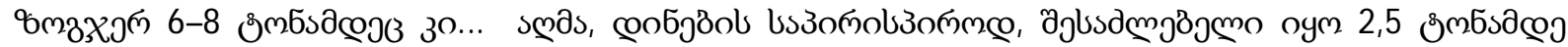

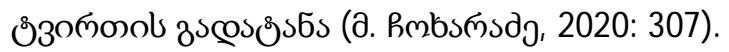

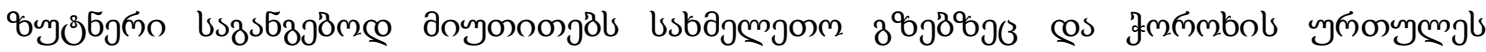

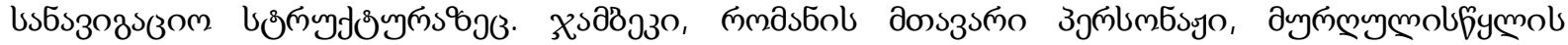

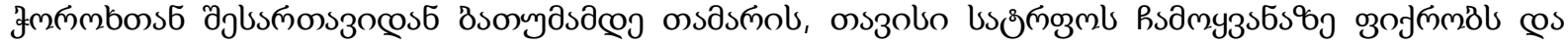

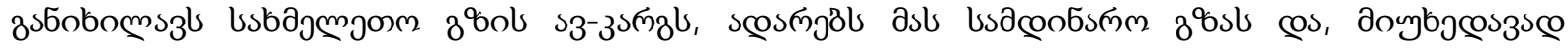

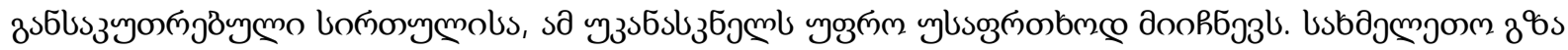

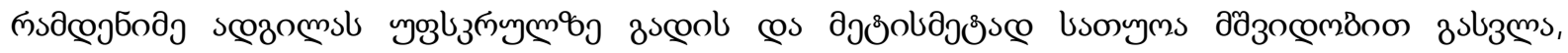

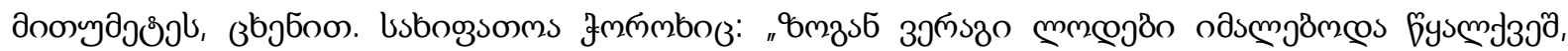

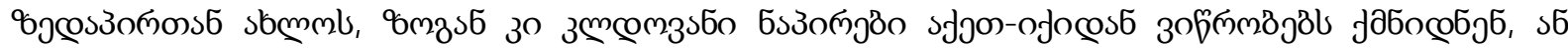

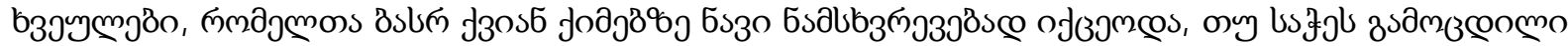

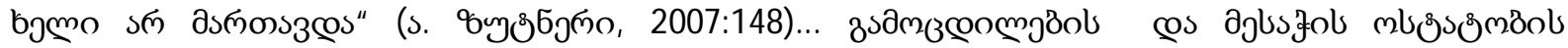

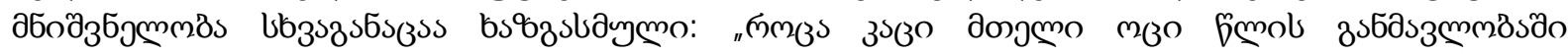

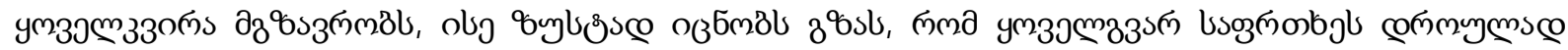

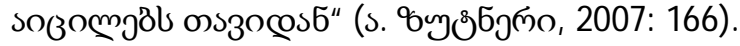

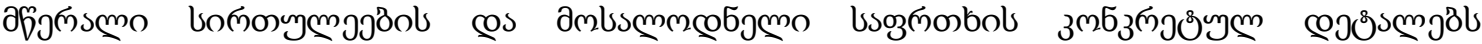

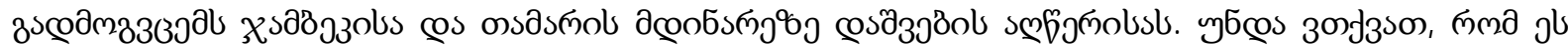

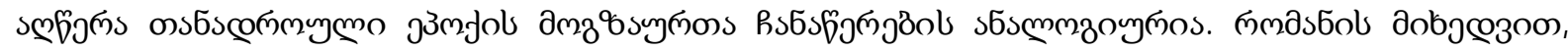

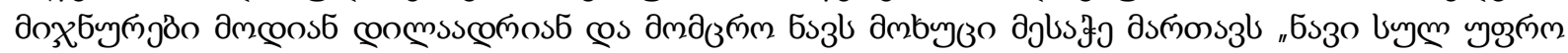

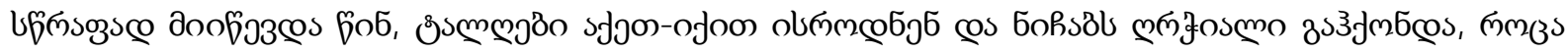




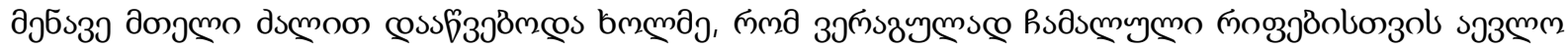

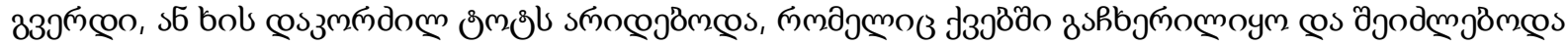

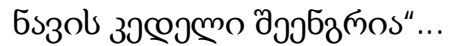

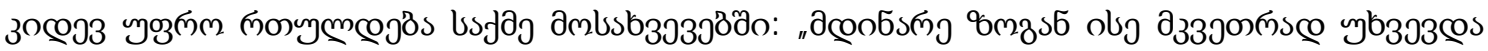

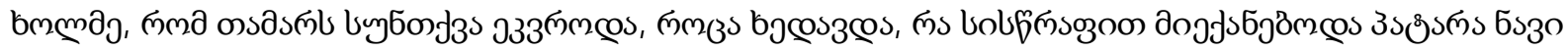

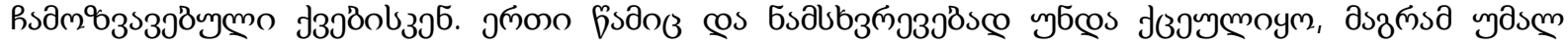

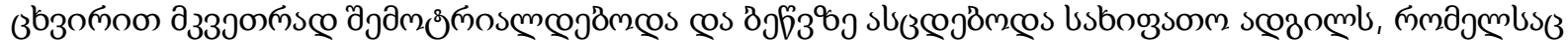

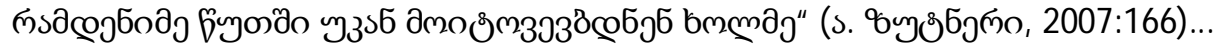

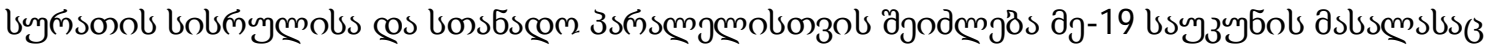

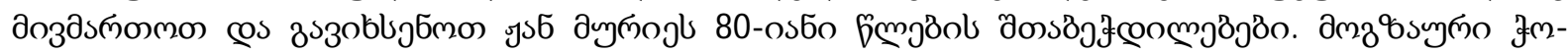

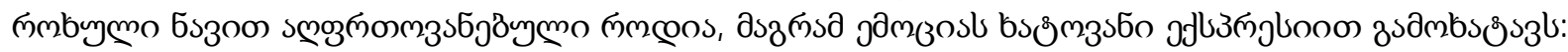

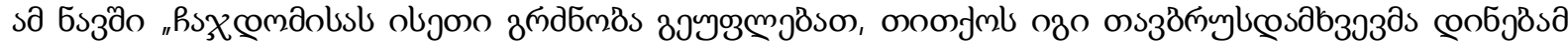

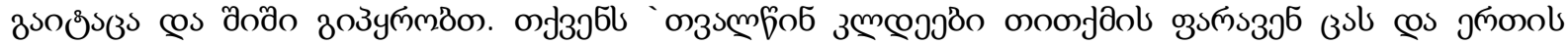

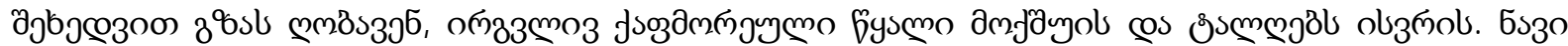

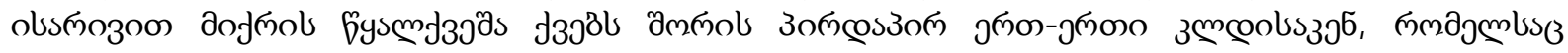

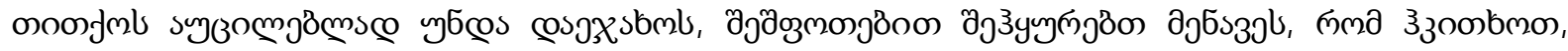

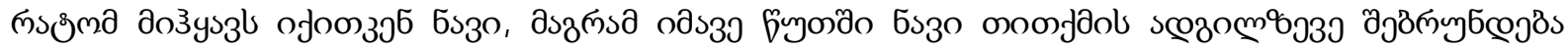

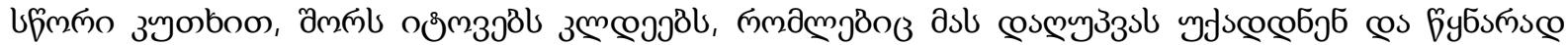

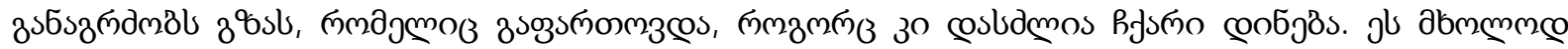

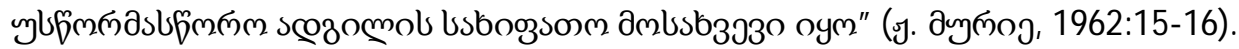

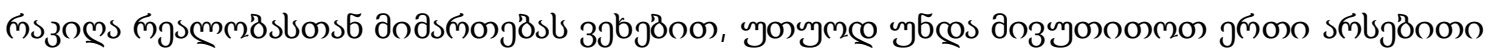

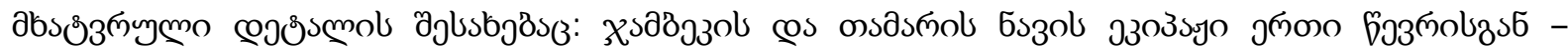

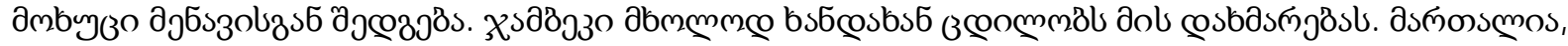

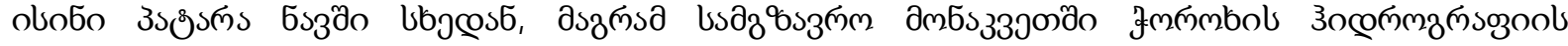

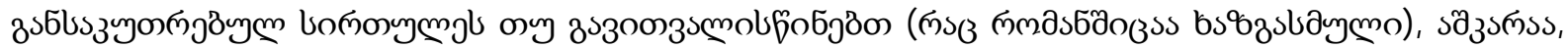

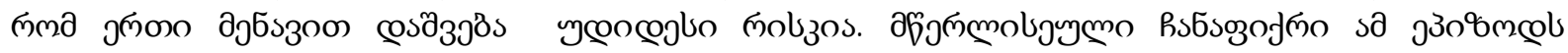

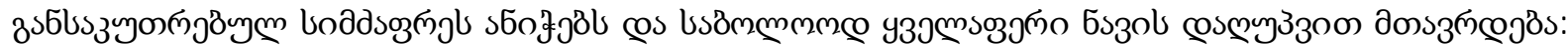

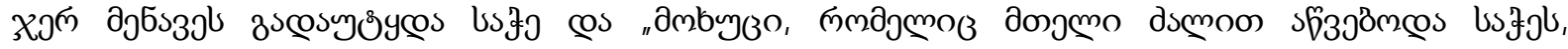

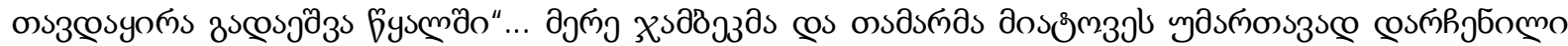

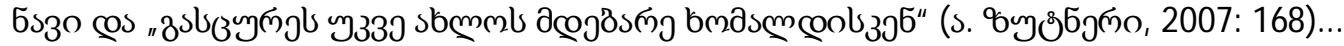

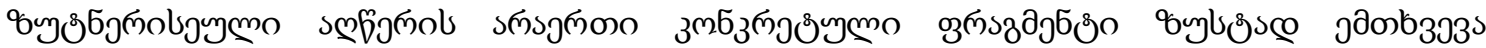

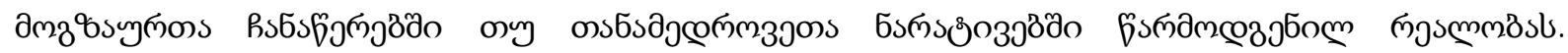

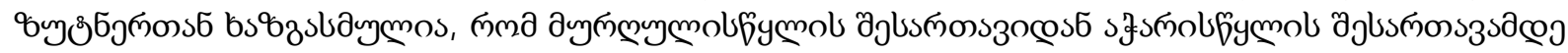

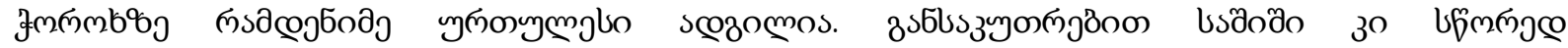

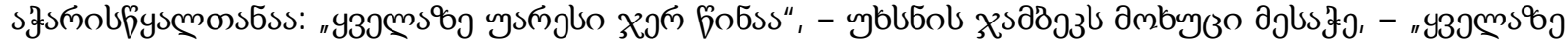

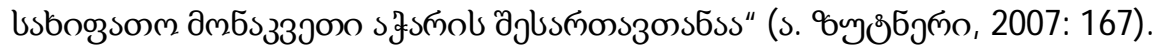

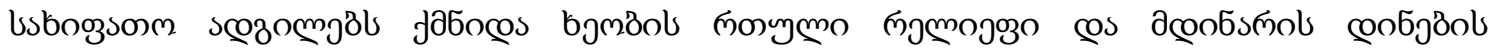

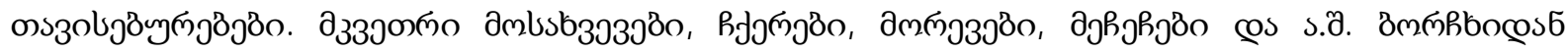

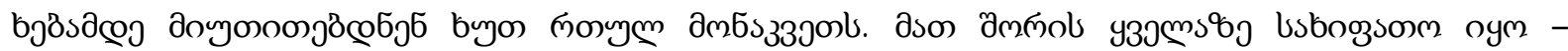

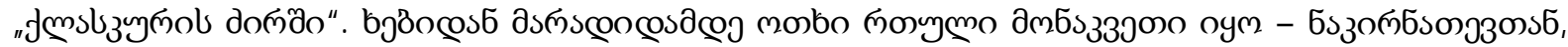

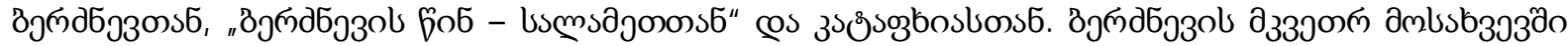

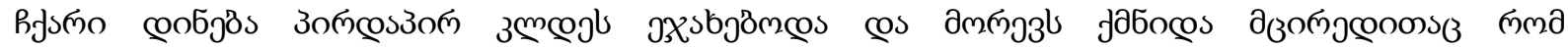

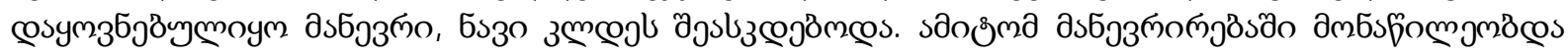

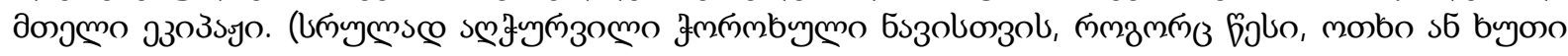

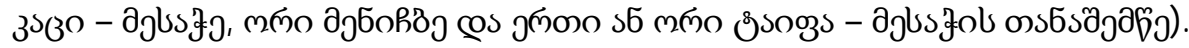

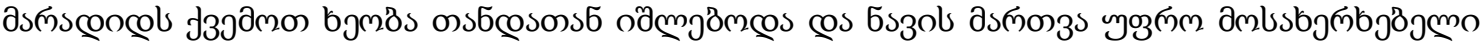

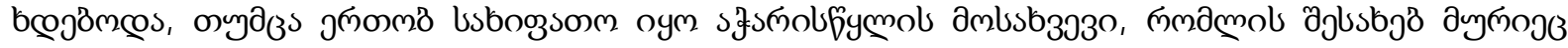

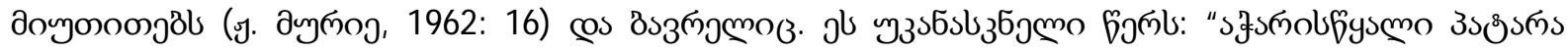

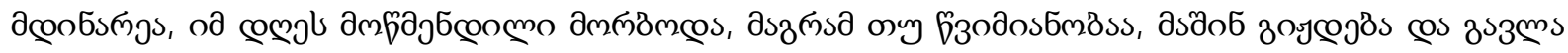

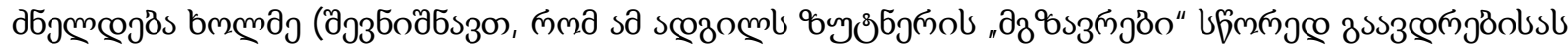




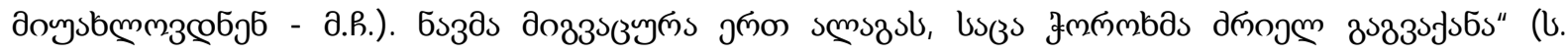

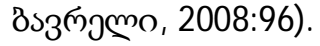

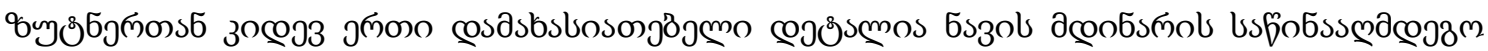

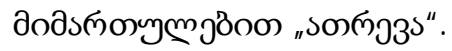

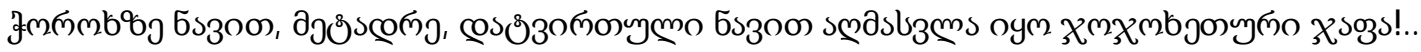

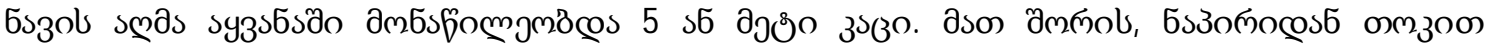

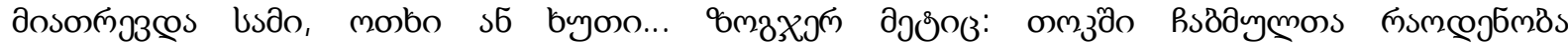

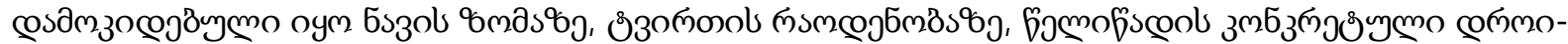

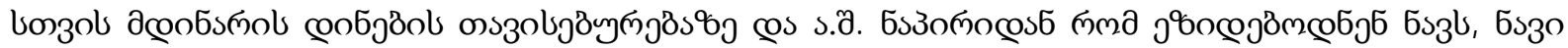

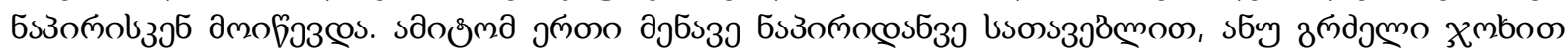

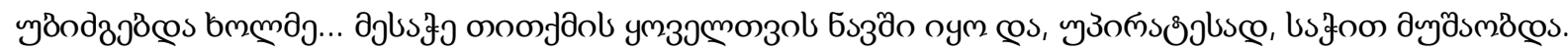

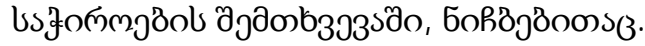

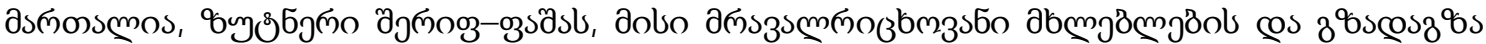

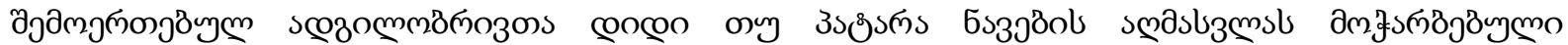

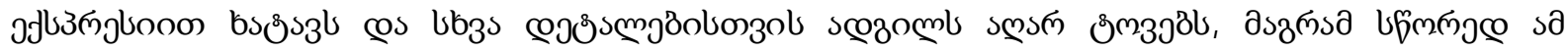

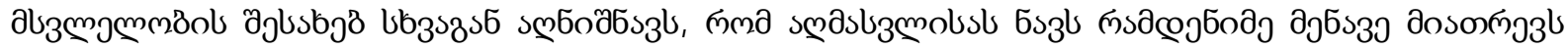

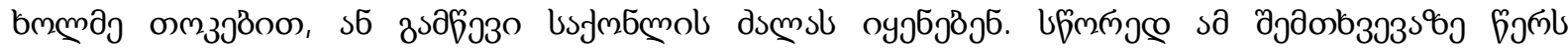

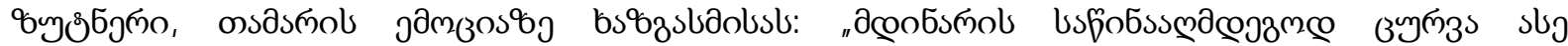

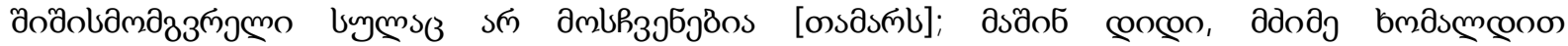

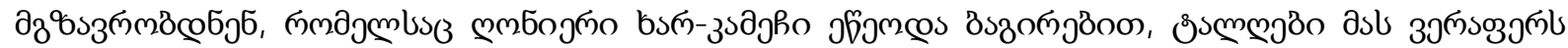

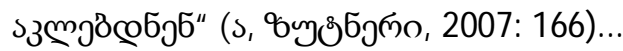

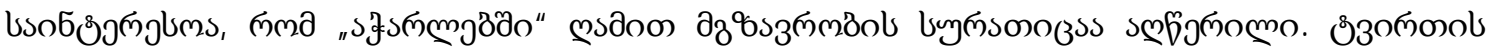

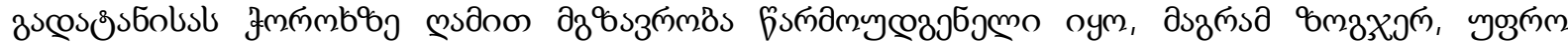

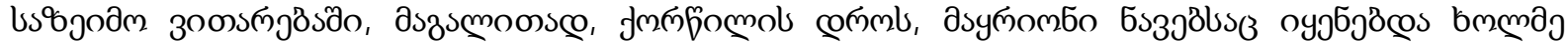

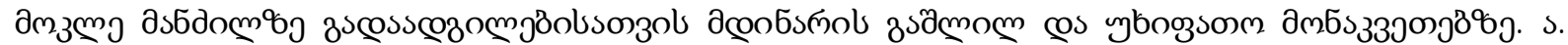

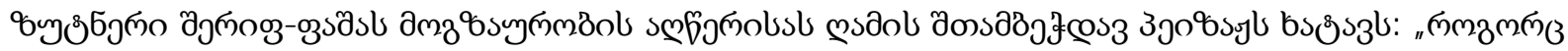

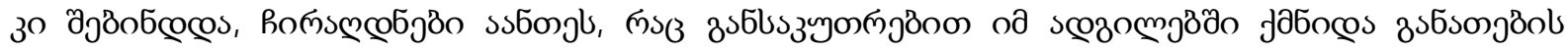

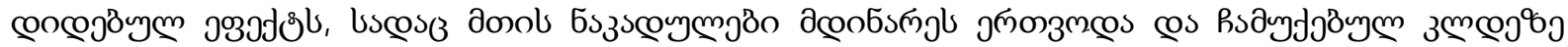

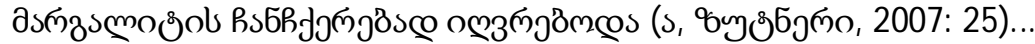

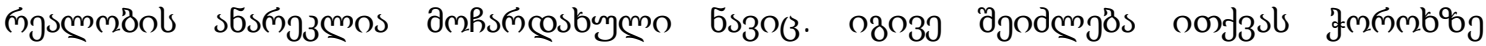

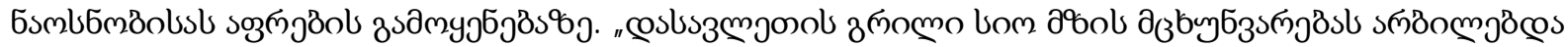

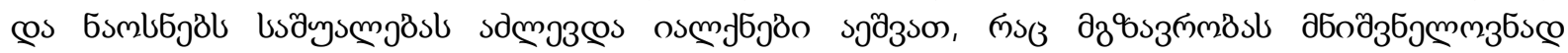

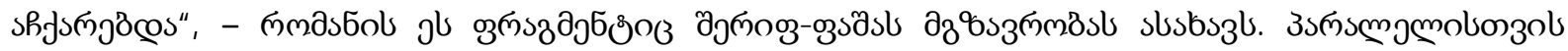

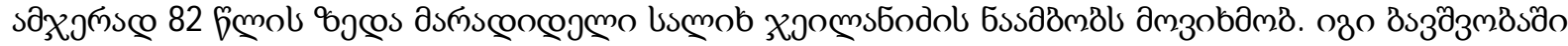

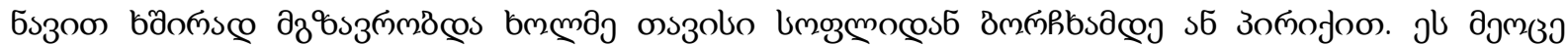

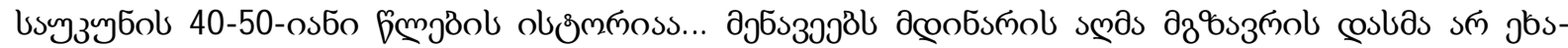

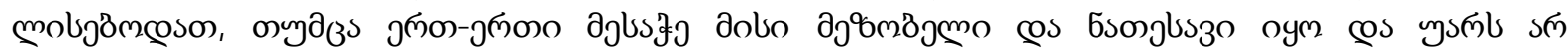

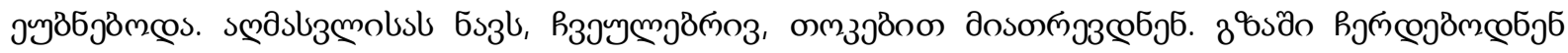

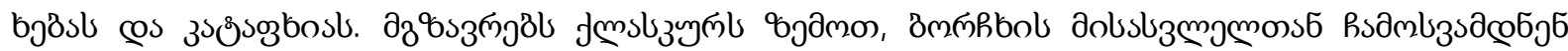

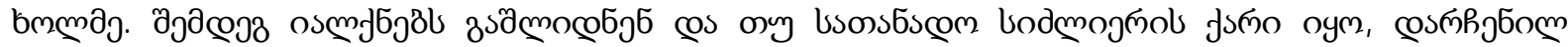

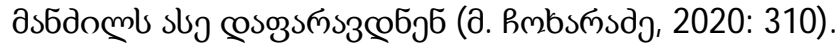

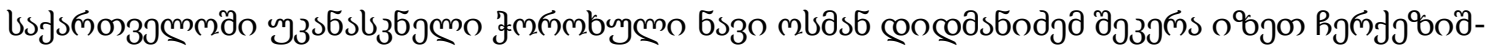

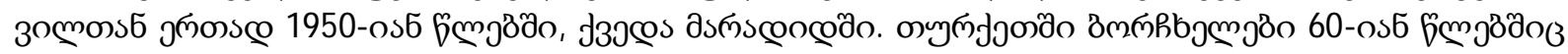

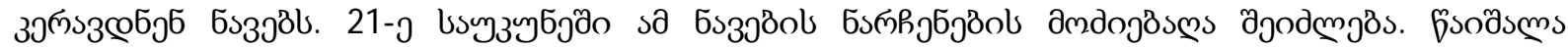

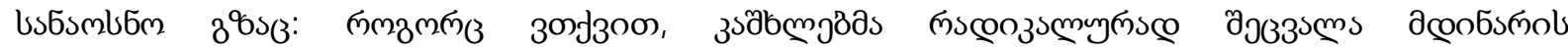

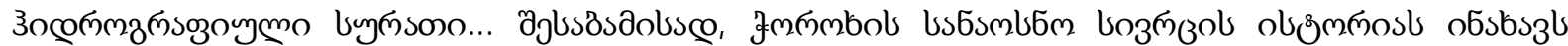

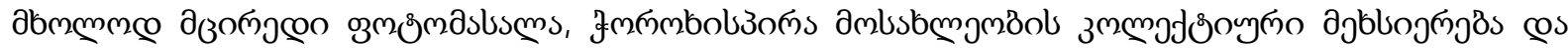

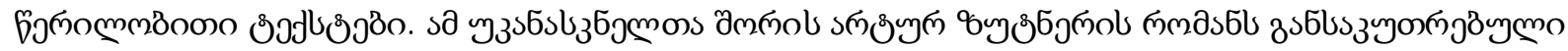

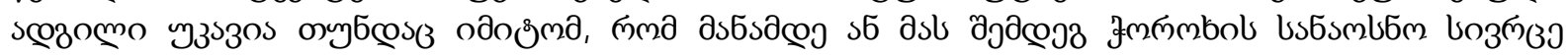

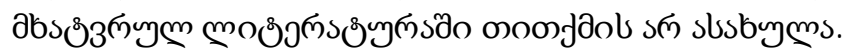




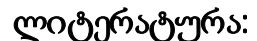

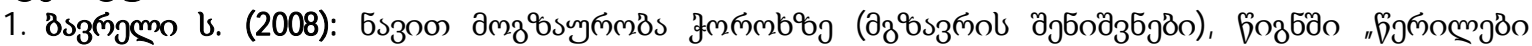

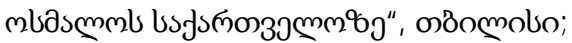

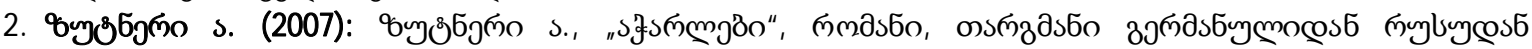

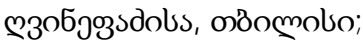

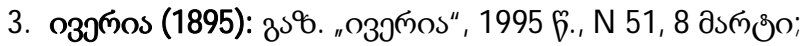

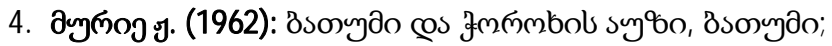

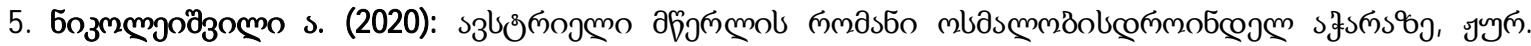
ұмпмьо, N 3, 2020 f., Вsомудо;

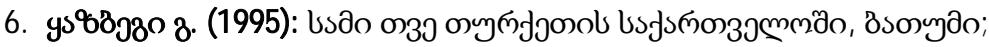

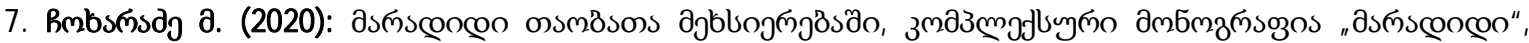

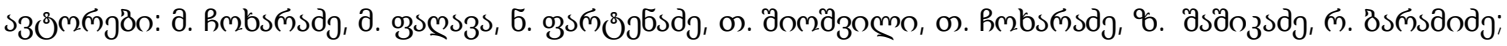

8. Aytekyn 0. (2013): Çoruh Irmağı Üzerinde Yapılan Tarihi Kayık Taşımacıllğı Üzerine Bir Değerlendirme, http://www.sosyal arastirmalar.com/cilt6/cilt6sayi25 pdf_ozelsayi/aytekin_osman.pdf

\section{Arthur Zuttner's Novel “Adjarians” and the Chorokhi Navigation Area}

\author{
Chokharadze Malkhaz \\ Sota Rustaveli State University, Batumi
}

\begin{abstract}
Chorokhi is the main river of historical southern Georgia. Interesting background knowledge about sailing on Chorokhi was preserved by the press and the records of travelers of the last quarter of the $19^{\text {th }}$ century and the beginning of the $20^{\text {th }}$ century, as well as by the collective memory of the population living by the edge of the river. Interestingly, the Chorokhi navigation area has almost never been reflected in fiction. Therefore, the work "Adjarians" by Arthur Zuttner is undoubtedly of great importance.

Arthur Zuttner, an Austrian writer, lived in Georgia with his wife Bertha from 1876-1885. "Adjarians" was published in Germany in 1888, and the Georgian translation by Rusudan Ghvinepadze was published in 2007.

"Adjarians" reflects the reality of southern Georgia before the Russo-Ottoman war of 187778. The action takes place mainly in the Chorokhi basin. The author describes the Chorokhi boat and sailing in only a few episodes, though, quite extensively. The work deals with the navigation images described by Zuttner in the novel, especially how the Chorokhi navigation area is reflected in the work and what is the relationship between the relevant episodes of the novel and the objective reality. It is obvious that the writer has deep insight into the life of the Chorokhi sailors and the local specifics of the river sailing. Obviously, Suttner's text is an artistic interpretation of reality, but it must also be noted that the navigational narrative in Suttner's novel, with a few exceptions, accurately depicts a number of $19^{\text {th }}$ century details of the Chorokhi navigation artery. Consequently, the images depicted in the novel are undoubtedly interesting for the literature lovers and the readers of fiction interested in the history or details of life.
\end{abstract}

Keywords: Zuttner, novel, Chorokhi, boat, sailing. 